\title{
Imaging Fundamental Electronic Excitations at High Spatial Resolution Using Scanning Cathodoluminescence Microscopy
}

\author{
M.R. Phillips*, D. Drouin**, S.J. Moody* and C. Ton-That*
}

* Microstructural Analysis Unit, University of Technology, Sydney PO Box 123 Broadway NSW Australia 2007

** Université de Sherbrooke, 2500 Bd de l'Université, J1K 2R1, Sherbrooke, Québec, Canada

Wide band gap semiconductors, such as $\mathrm{ZnO}, \mathrm{GaN}, \mathrm{ZnS}$ and $\mathrm{CdS}$, exhibit free-exciton (FE) cathodoluminescence (CL) emission at room temperature due to their relatively large exciton binding energies. Using a SEM operating a low voltage $(<2 \mathrm{kV})$ this CL signal can be used to image and analyse the propagation of fundamental electronic excitations at nano-scale spatial resolution in bulk and nano-structured materials, particularly energy-transport in individual nano-wires.

$\mathrm{CL}$ images of $\mathrm{ZnO}$ nanowires using the FE emission generally exhibit a bright-end as well as banding along their length (Fig. 1), which has been attributed to the radiative decay of excitonpolaritons $[1,2]$. These mixed state quasi-particles result from strong coupling between photon and exciton electromagnetic fields, and radiatively decay following scattering by the electric fields induced from lattice vibrations. A typical CL spectrum consisting of the FE peak and its longitudinal-optical (LO) phonon replicas is shown in Fig. 2. Monochromatic CL images of nonradiative extended dislocations in bulk c-axis $\mathrm{ZnO}$ crystals using the $\mathrm{FE}$ and FE-1LO CL emission are shown in Fig. 3 [3]. A 2kV accelerating voltage and sub $2 \mathrm{~nm}$ electron probe were used to provide an electron-solid interaction volume approaching that of a point source. CL data was collected at liquid nitrogen and room temperature. The latter was used to maximize radiative FE decay by thermally ionizing donors involved in bound exciton and free-to-bound recombination channels. The CL dislocation images in Fig. 3 reveal excitonic spatial spreading of around $200 \mathrm{~nm}$ with this distance being clearly greater for the FE emission compared with the FE-1LO peak. This behavior is attributed to FE emission being reabsorbed by exciton formation, extending the effective FE diffusion length compared with the FE-1LO. This photon-recycling effect is not observed with the FE-1LO emission since its energy is below the band gap.

The bright ends observed in $\mathrm{ZnO}$ nanowires fabricated in this work are typically observed for rods with diameters below $\sim 200 \mathrm{~nm}$, which is consistent with the short exciton diffusion length observed in bulk material. In addition, this increase in CL at the end of the wire is observed in both FE and FE-1LO CL images. Individual nanowires dispersed on a holey carbon support film were also investigated by STEM and STEM-CL image modes Comparison of equivalent STEM, conventional CL and STEM-CL images (not shown) suggest that the CL contrast variation along the axis of the nanowire in our case relates to subtle changes in rod thickness along its length rather than polariton interference effects.

\section{References}

[1] Wires grown by the vapour-liquid-solid (VLS) method using Au nanodots catalysts on (11-20) sapphire substrates, see M. Foley, Appl. Phys. Lett. 93, 243104 (2008)

[2] L.K. van Vugt, Phys. Rev. Lett. 97, 147401 (2006)

[3] ZnO (0001) single crystals obtained from MTI Corporation, Richmond, CA 


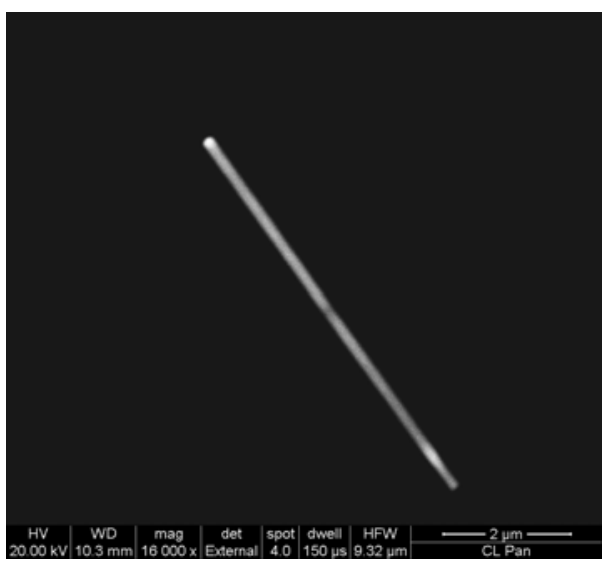

Figure 1 Panchromatic CL image of a $\mathrm{ZnO}$ nanowire grown by the VLS method.

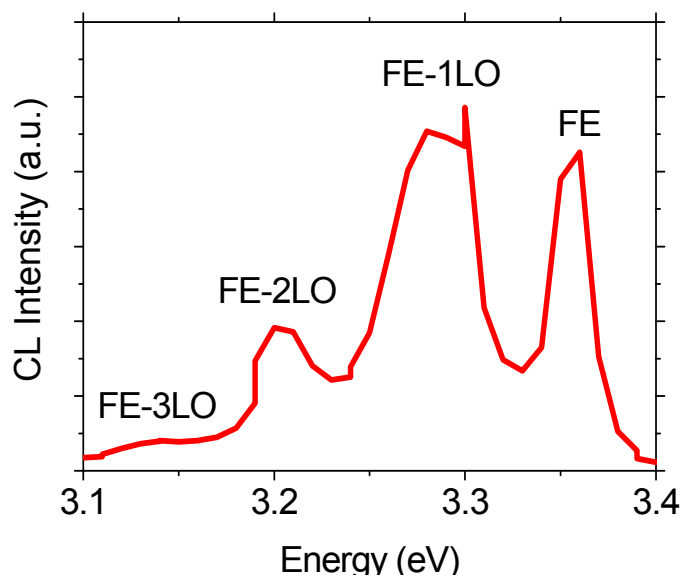

Fig. 2 Typical CL spectrum consisting of the FE peak and its phonon replicas.

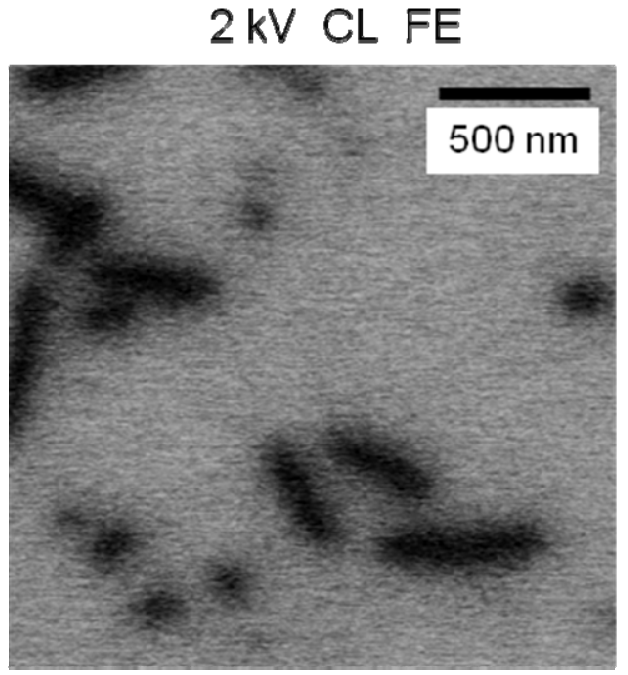

$2 \mathrm{kV} C L$ FE - 1LO

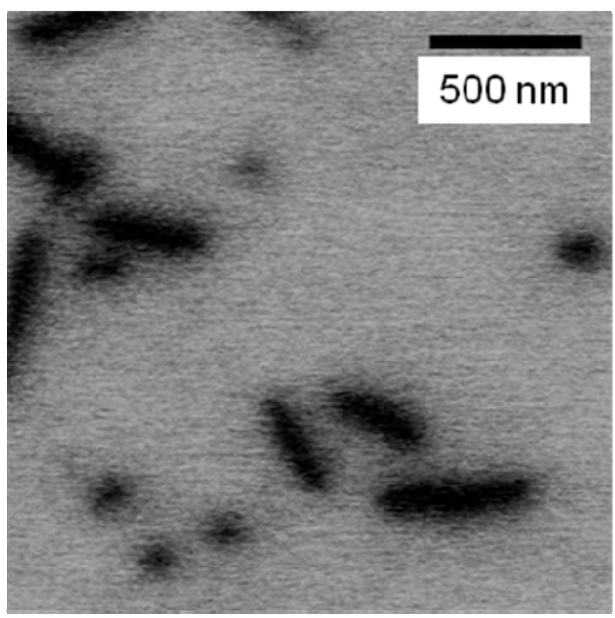

Fig. 3 Monochromatic CL of non-radiative dislocations in bulk c-axis $\mathrm{ZnO}$. 